\section{Combination Therapy with Methotrexate and Etanercept for Refractory Chronic Recurrent Multifocal Osteomyelitis}

\section{To the Editor:}

Chronic recurrent multifocal osteomyelitis (CRMO) is a form of chronic nonbacterial osteitis $(\mathrm{CNO})$ characterized by one or more lytic bone lesions with no identifiable cause ${ }^{1}$. The metaphyses of long bones and the clavicle are most frequently affected, although any bone may be involved. Most cases begin in childhood, and follow an intermittent course. Pain is the most common presenting symptom. Affected individuals are sometimes febrile, and acute-phase reactants may be increased, pointing to the inflammatory expression of this condition. Lesions may resolve at or before the time of puberty, but in some cases skeletal deformity and/or disability may occur $^{2}$.

We describe 2 children with CRMO whose symptoms persisted after trials of nonsteroidal antiinflammatory medication, glucocorticoids, and methotrexate (MTX). Combination therapy with MTX and etanercept resulted in amelioration of pain, accompanied in one patient by radiographic improvement in a lytic bone lesion.

Case 1. A 4-year-old boy developed pain in his right foot and refused to walk for 2 months. Plain radiographs showed normal-appearing vertebra. A bone scan disclosed increased uptake in the right talus and navicular bone. He received several courses of intravenous and oral antimicrobials, without improvement. Fever was absent, C-reactive protein was normal, and erythrocyte sedimentation rate (ESR) was mildly increased to 23 $\mathrm{mm} / \mathrm{h}$. A second bone scan performed 2 months later showed increased radionuclide uptake in the $\mathrm{L} 4$ and $\mathrm{L} 5$ vertebra. Focal magnetic resonance imaging (MRI) revealed vertebral abnormalities consistent with CRMO including increased signal intensity of the vertebral marrow (Figure 1A). Talar biopsy was nonrevealing, showing bone fragments with myxoidappearing fibrous stroma. Naproxen $10 \mathrm{mg} / \mathrm{kg} / \mathrm{day}$ was given, without improvement. Prednisone $2 \mathrm{mg} / \mathrm{kg} /$ day was added for 3 weeks, with reduction in pain, but symptoms recurred during taper. During the third month, oral MTX (15 mg/m ${ }^{2}$ once weekly) was given with naproxen. However, he complained of worsening leg and back pain (verbally described as 7 on a scale of 1-10) and was unable to walk. Etanercept $0.8 \mathrm{mg} / \mathrm{kg} / \mathrm{week}$ was prescribed with MTX and naproxen. One month later, his limp had resolved completely, and he reported no pain. ESR was $16 \mathrm{~mm} / \mathrm{h}$. MRI performed after 6 months of treatment showed diminished signal abnormality, indicating improvement in bone inflammation (Figure 1B), and he remained symptom-free. His care was transferred to another medical center.

Case 2. A 12-year-old girl presented with elbow, ankle, hip, and foot pain worsened by activity for 6 months, without fever. Radiographs disclosed numerous lucent bony lesions with sclerotic margins in the metaphyses of the left distal tibia and fibula, and a healing fracture in the distal portion of the right fourth metatarsal. Focal MRI showed lesions with enhanced T2 signal including pelvic, right ankle, and left foot lesions, as well as in the acetabular roof, pubic rami, second sacral segment, distal tibia and fibula, and fourth and fifth metatarsal bones. Direct biopsy was not performed at request of the family. However, the clinical and radiographic findings were compatible with presumed $\mathrm{CRMO}^{3}$. A bone marrow aspirate showed normal cytology. Naproxen $10 \mathrm{mg} / \mathrm{kg} /$ day, prednisone $(2 \mathrm{mg} / \mathrm{kg} /$ day for 1 month, followed by slow taper), and oral MTX $\left(10 \mathrm{mg} / \mathrm{m}^{2}\right.$ once weekly) were given in succession. However, after 8 months, she complained of right buttock, hip, and left ankle pain (described as 4 on a scale of 1-10) and limp. Etanercept $(0.4 \mathrm{mg} / \mathrm{kg} / \mathrm{dose}$ subcutaneously twice weekly) was added to MTX. Within several weeks, her pain resolved completely, and ESR declined from 18 to $8 \mathrm{~mm} / \mathrm{h}$. Pain did not recur during 15 months of treatment. Because the patient felt well, her family declined followup MRI studies.

We describe 2 children with CRMO who continued to experience pain despite antiinflammatory treatment using naproxen, glucocorticoids, and MTX. However, coadministration of MTX and etanercept, a tumor necrosis factor- $\alpha$ (TNF- $\alpha$ ) blocker, resulted in progressive and sustained improvement in pain. In one child, clinical improvement was associated with radiographic improvement in a bone lesion. A limitation of our report is that in accord with the parents' wishes, posttreatment MRI was not performed until several months after therapy with etanercept was started. Since the natural history of CRMO may include spontaneous remission, we cannot exclude the possibility that MRI changes over this interval may have been incidental rather than due to etanercept treatment.

Up to $25 \%$ of cases of CRMO are accompanied by inflammation involving other organs, including the skin or bowel ${ }^{2}$. Histologic examination of bone lesions may reveal neutrophilic inflammation with increased osteoclasts ${ }^{4}$. These observations suggest that CRMO may be an autoinflammatory disorder. Osteolytic lesions resembling those of CRMO are seen in infants genetically deficient in expression of a natural soluble interleukin 1 receptor antagonist (DIRA). However, unlike CRMO, infants affected with DIRA also exhibit other skeletal abnormalities including generalized osteopenia, suggesting the mechanisms underlying bone pathology in these 2 disorders may not be identical ${ }^{5,6}$. Majeed syndrome, a syndromic form of CRMO associated with dyserythropoietic anemia, is caused by mutations in LPIN2 ${ }^{7}$.

There is evidence for a possible role of the proinflammatory cytokine TNF- $\alpha$ in the pathogenesis of CNO. In a large registry, serum concentrations of TNF- $\alpha$ were increased in two-thirds of patients with active disease $^{1}$. Increased TNF- $\alpha$ expression was observed in biopsies obtained from osteolytic lesions of 2 adults with SAPHO (synovitis, acne, pustulosis, hyperostosis, and osteitis) syndrome, a disorder that presents mainly in adulthood and produces bony lesions similar to those of CRMO ${ }^{8}$. TNF- $\alpha$ promotes osteoclast activation in vitro, representing a possible mechanism by which local TNF- $\alpha$ overexpression may contribute to osteolysis ${ }^{9}$.

Etanercept is a recombinant, humanized dimeric protein containing the extracellular domain of the human $\mathrm{p} 75 \mathrm{TNF}$ receptor fused to the $\mathrm{Fc}$ region

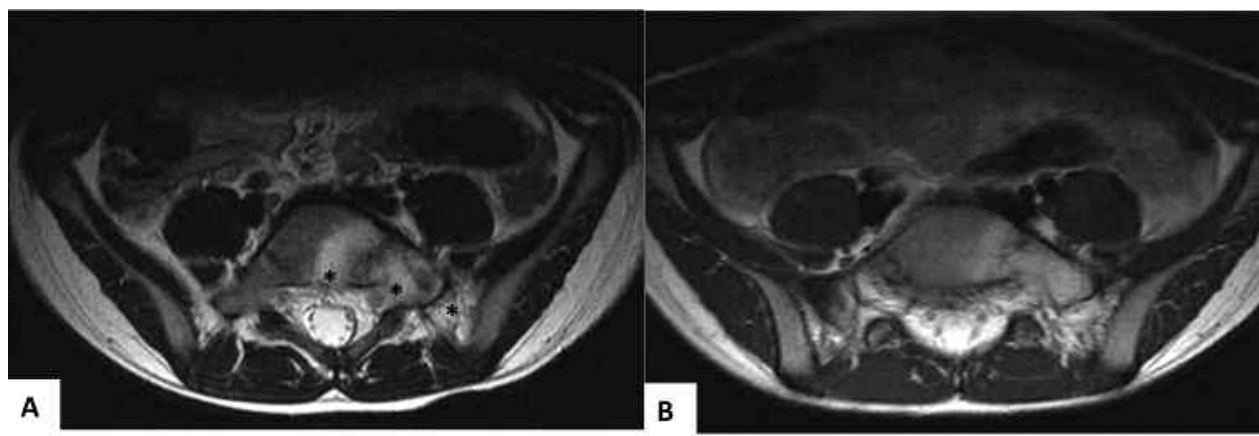

Figure 1. MRI images of Case 1 before (A) and after (B) anti-TNF therapy. (A) *Multifocal areas of enhancement involving the sacrum and lower aspect of L5 vertebral body as well as iliac wings. (B) Resolving edema of S1/L5 vertebral bodies. All images are T2-weighted post-gadolinium. 
of $\operatorname{IgG1}$, which antagonizes soluble TNF- $\alpha$. Etanercept was given with MTX in our cases, based on favorable experience using combined therapy with these drugs in juvenile idiopathic arthritis ${ }^{10}$. There are several reports of successful use of infliximab, a chimeric anti-TNF- $\alpha$ antibody, in CRMO and SAPHO, one report on the use of etanercept in SAPHO, and one report of use of the anti-TNF- $\alpha$ antibody adalimumab to treat CRMO ${ }^{11,12,13}$. To our knowledge, this is the first description of successful use of etanercept with MTX in children with CRMO.

Bisphosphonates have also been used successfully for cases of CRMO that did not respond to initial therapy ${ }^{14,15,16}$. We chose etanercept for our patients because of concern regarding the longterm effects of bisphosphonates on the growing skeleton, and reports of osteonecrosis of the jaw associated with their use in adults. Therapy with TNF- $\alpha$ antagonists may be complicated by hypersensitivity reactions or by infection. In addition, recent data have emerged questioning the longterm safety of these agents in children with respect to risk for malignancy. However, the overall risk for cancer in children receiving TNF- $\alpha$ antagonists for rheumatic disease appears to be quite low ${ }^{17,18}$.

To date, no specific therapy has been universally efficacious for CNO: treatment failures of bisphosphonates have been reported, and incomplete responses to infliximab or waning efficacy over time have been described $^{12}$. Further, since biologically significant differences exist between different TNF- $\alpha$ antagonists ${ }^{19}$, it cannot be assumed that drugs of this class are interchangeable. A multicenter randomized, controlled trial with predetermined outcome measures comparing efficacy of etanercept with MTX, an anti-TNF- $\alpha$ antibody such as infliximab, and a bisphosphonate in $\mathrm{CRMO} / \mathrm{CNO}$ refractory to initial therapy might provide information useful to clinicians caring for children with this condition. At present, we believe that TNF- $\alpha$ antagonists or bisphosphonates are both acceptable treatment alternatives. Whichever class of drug is chosen, we further believe that before treatment is commenced, the known risks and potential benefits should be explained to patients and their families and their consent should be obtained. In cases when treatment with a TNF- $\alpha$ antagonist is contemplated, etanercept with MTX may be preferred to infliximab, since the former regimen can be given at home rather than by intravenous infusion, and immediate adverse reactions are less likely.

ELI M. EISENSTEIN, MD, Attending Pediatrician, Department of Pediatrics, Hadassah-Hebrew University Medical Center, Mount Scopus, Jerusalem, Israel; GRANT D. SYVERSON, MD, Instructor; SHEETAL S. VORA, MD, Assistant Professor, Medical College of Wisconsin, Children's Research Institute; CALVIN B. WILLIAMS, MD, PhD, D.B. and Marjorie Reinhart Chair in Rheumatology, Children's Hospital of Wisconsin; Associate Professor, Pediatrics, Medical College of Wisconsin, Children's Research Institute, Milwaukee, Wisconsin, USA. Address correspondence to Dr. Eisenstein;

E-mail: emeisenstein@gmail.com

\section{REFERENCES}

1. Jansson A, Renner ED, Ramser J, Mayer A, Haban M, Meindl A, et al. Classification of non-bacterial osteitis: retrospective study of clinical, immunological and genetic aspects in 89 patients. Rheumatology 2007;46:154-60.

2. El-Shanti HI, Ferguson PJ. Chronic recurrent multifocal osteomyelitis: a concise review and genetic update. Clin Orthop Relat Res 2007;462:11-9.

3. Jansson AF, Müller TH, Gliera L, Ankerst DP, Wintergerst U, Belohradsky BH, et al. Clinical score for nonbacterial osteitis in children and adults. Arthritis Rheum 2009;60:1152-9.

4. Bjorkstén B, Boquist L. Histopathological aspects of chronic recurrent multifocal osteomyelitis. J Bone Joint Surg Br 1980;62:376-80.
5. Reddy S, Jia S, Geoffrey R, Lorier R, Suchi M, Broeckel U, et al. An autoinflammatory disease due to homozygous deletion of the IL1RN locus. N Engl J Med 2009;360:2438-44.

6. Aksentijevich I, Masters SL, Ferguson PJ, Dancey P, Frenkel J, van Royen-Kerkhoff A, et al. An autoinflammatory disease with deficiency of the interleukin-1-receptor antagonist. N Engl J Med 2009;360:2426-37.

7. Ferguson PJ, Chen S, Tayeh MK, Ochoa L, Leal SM, Pelet A, et al. Homozygous mutations in LPIN2 are responsible for the syndrome of chronic recurrent multifocal osteomyelitis and congenital dyserythropoietic anaemia (Majeed syndrome). J Med Genet 2005;42:551-7.

8. Hurtado-Nedelec M, Chollet-Martin S, Nicaise-Roland P, Grootenboer-Mignot S, Ruimy R, Meyer O, et al. Characterization of the immune response in the synovitis, acne, pustulosis, hyperostosis, osteitis (SAPHO) syndrome. Rheumatology 2008;47:1160-7.

9. Kobayashi K, Takahashi N, Jimi E, Udagawa N, Takami M, Kotake $\mathrm{S}$, et al. Tumor necrosis factor alpha stimulates osteoclast differentiation by a mechanism independent of the ODF/RANKL-RANK interaction. J Exp Med 2000;191:275-86.

10. Horneff G, De Bock F, Foeldvari I, Girschick HJ, Michels H, Moebius D, et al. Safety and efficacy of combination of etanercept and methotrexate compared to treatment with etanercept only in patients with juvenile idiopathic arthritis (JIA): preliminary data from the German JIA Registry. Ann Rheum Dis 2009;68:519-25.

11. Deutschmann A, Mache CJ, Bodo K, Zebedin D, Ring E. Successful treatment of chronic recurrent multifocal osteomyelitis with tumor necrosis factor-alpha blockage. Pediatrics 2005;116:1231-3

12. Eleftheriou D, Gerschman T, Sebire N, Woo P, Pilkington CA, Brogan PA. Biologic therapy in refractory chronic non-bacterial osteomyelitis of childhood. Rheumatology 2010;49:1505-12.

13. Moll C, Hernández MV, Cañete JD, Gómez-Puerta JA, Soriano A, Collado A, et al. Ilium osteitis as the main manifestation of the SAPHO syndrome: response to infliximab therapy and review of the literature. Semin Arthritis Rheum 2008;37:299-306.

14. Simm PJ, Allen RC, Zacharin MR. Bisphosphonate treatment in chronic recurrent multifocal osteomyelitis. J Pediatr 2008; 152:571-5.

15. Gleeson H, Wiltshire E, Briody J, Hall J, Chaitow J, Sillence D, et al. Childhood chronic recurrent multifocal osteomyelitis: pamidronate therapy decreases pain and improves vertebral shape. J Rheumatol 2008;35:707-12.

16. Miettunen PM, Wei X, Kaura D, Reslan WA, Aguirre AN, Kellner JD. Dramatic pain relief and resolution of bone inflammation following pamidronate in 9 pediatric patients with persistent chronic recurrent multifocal osteomyelitis (CRMO). Pediatr Rheumatol Online J 2009;7:2.

17. McCroskery P, Wallace CA, Lovell DJ, Stryker S, Chernyukhin N, Blosch C, et al. Summary of worldwide pediatric malignancies reported after exposure to etanercept. Pediatr Rheumatol Online $\mathbf{J}$ 2010;8:18.

18. Cron RQ, Beukelman T. Guilt by association - what is the true risk of malignancy in children treated with etanercept for JIA? Pediatr Rheumatol Online J 2010;8:23.

19. Mitoma H, Horiuchi T, Tsukamoto H, Tamimoto Y, Kimoto Y, Uchino A, et al. Mechanisms for cytotoxic effects of anti-tumor necrosis factor agents on transmembrane tumor necrosis factor alpha-expressing cells: comparison among infliximab, etanercept, and adalimumab. Arthritis Rheum 2008;58:1248-57.

J Rheumatol 2011;38:4; doi:10.3899/jrheum.100431 